\title{
Cómo vivir con EPOC: percepción de los pacientes
}

\author{
Francisca Muñoz-Cobos ${ }^{*}$, Nieves Acero-Guasch ${ }^{2}$, Rebeca Cuenca-del-Moral ${ }^{3}$, Pilar Barnestein-Fonseca ${ }^{4}$, Francisca Leiva- \\ Fernández $z^{4}$ y Antonio García-Ruiz ${ }^{5}$ \\ 1Centro de Salud El Palo. Distrito Sanitario Málaga (España). \\ 2 Area de Salud de Ibiza y Formentera (España). \\ 3 Centro de Salud Albarizas. Distrito Sanitario Costa del Sol (España). \\ 4 Unidad Docente Multiprofesional de Atención Familiar y Comunitaria. Distrito Sanitario Málaga (España). \\ 5Departamento Farmacología y Terapéutica. Facultad de Medicina. Universidad de Málaga (España).
}

\begin{abstract}
Resumen: Este estudio pretende conocer la representación mental sobre la Enfermedad Pulmonar Obstructiva Crónica (EPOC) que tienen los pacientes que la presentan, siguiendo el Modelo de Autorregulación de Sentido Común de Leventhal. Para ello llevamos a cabo un estudio exploratorio descriptivo mediante metodología cualitativa. Incluimos pacientes diagnosticados de EPOC atendidos en 3 centros de salud urbanos y 1 rural. Utilizamos la entrevista grupal video-grabada y analizamos el contenido utilizando el programa Atlas.Ti. 5.2. Realizamos 22 entrevistas grupales (20092011) incluyendo 58 pacientes y 3 sesiones de validación del informe por los participantes. Los resultados muestran que no existe un modelo mental compartido de la EPOC: la enfermedad tiene poco reconocimiento como tal; se diagnostica en descompensaciones y se confunde con otras enfermedades; la causa se atribuye no sólo al tabaco sino a "algo más" (humos, resfriados, trabajos duros); se minimizan los síntomas, incluyendo también los no respiratorios (dolor, cansancio) y se confunden con los de otras enfermedades. Existen creencias erróneas sobre los "resfriados", efecto del "fumar poco" y efecto "nocivo" de los inhaladores. Aparecen sentimientos de desesperanza, resignación y miedo. Concluimos que los pacientes con EPOC manifiestan confusión en muchos aspectos de la enfermedad mostrando dificultades para identificar un modelo mental sobre ella.

Palabras clave: Enfermedad pulmonar obstructiva crónica; Cognición; Investigación cualitativa.
\end{abstract}

\section{Introducción}

La Enfermedad Pulmonar Obstructiva Crónica (EPOC) se caracteriza por una obstrucción no reversible al flujo aéreo, cuyo principal factor etiológico es el consumo de tabaco y que ocasiona una dificultad progresiva para respirar, ocasionando importantes limitaciones a las personas que las padecen. Su prevalencia entre los 40 y 80 años en nuestro país se sitúa en torno a 10.2\% (Miravitlles et al., 2009) siendo muy importante el infradiagnóstico de esta enfermedad (Miravitlles et al., 2006).

En la actualidad se considera la EPOC como un trastorno multicomponente (Soler-Cataluña, Martínez-García y Catalán, 2010) y existen distintos fenotipos clínicos de la enfermedad, como múltiples "pequeñas EPOC" (Renard y Vestbo, 2008) con historia natural y requerimientos de tratamiento diferentes (Han et al., 2010), existiendo una amplia variabilidad entre los pacientes que la presentan. Además existen importantes discrepancias entre las medidas objetivas de parámetros funcionales de la EPOC y la percepción subjetiva de los pacientes, lo que supone la necesidad de evaluar

* Dirección para correspondencia [Correspondence address]:

Francisca Muñoz Cobos. Avda Moliere 25, esc-2 5º-3. 29004, Málaga (España). E-mail: franciscam@ono.com

\section{Title: How to live with COPD: patient's perception.}

Abstract: The aim of this study is to determine the mental model that patients with COPD (chronic obstructive pulmonary disease) have about their illness analyzed by the Leventhal's Common Sense of Self-Regulation Model. An exploratory-descriptive study using qualitative methodology was performed. We included COPD-diagnosed patients attending three urban and a rural primary care centres. We used video-taped group interviews and transcribed them using Atlas.Ti 5.2 program. 22 group interviews (2009 2011) and 58 patients were included. There were 3 additional sessions to final inform validation. The results show that patients did not have a defined mental model about COPD: this disease was insufficiently recognized; diagnosis was made in exacerbations and it was confused with other diseases; the cause of illness was related to tobacco consumption and to "something else": fumes, colds and hard work. Patients tended to minimize their symptoms and limitations. Participants had wrong beliefs about colds, smoking only a few cigarettes effects and damage of inhaled treatment. Emotions as despair, resignation and fear were reported. We conclude that COPD patients showed confusion about many aspects of the disease (causes, symptoms, treatments). It was difficult to identify a mental model of the disease among COPD patients.

Key words: Chronic Obstructive Pulmonary Disease; Cognition; Qualitative Research.

la afectación producida por la enfermedad de un modo individual.

Las actividades más afectadas abarcan aspectos variados como la actividad física, ocio y sexualidad (ÁlvarezGutiérrez et al., 2007), con mayor impacto en pacientes de mayor edad y menor nivel cultural y económico (De la Iglesia et al., 2001). La identificación y manejo de los signos y síntomas físicos de la EPOC se ha incrementado con el conocimiento de la enfermedad; sin embargo las repercusiones psicosociales son menos reconocidas e investigadas.

El estudio de los aspectos subjetivos de la enfermedad se ha centrado en la valoración de la Calidad de Vida Relacionada con la Salud (CVRS) medida mediante instrumentos, entre los que destacan el cuestionario St George (Ferrer et al., 1996) y el Quality of life for respiratory illness questionnaire (QOLRIQ) (Maillé et al., 1997). Además de estas medidas, existe interés creciente por la valoración de las consecuencias de la enfermedad referidas por el propio paciente, los denominados patient-reported outcomes (PRO), centrándose en actividades de la vida diaria (Miravitlles, Anzueto, Legnani, Forstmeier y Fargel, 2007), estado de ánimo (Maurer et al., 2008), síntomas psicoemocionales y relaciones sociales (Boult y Wieland, 2010).

Asimismo es fundamental la valoración de las creencias de los pacientes, ya que se sabe que existe relación entre me- 
jor calidad de vida y creencias más positivas acerca de la enfermedad (Sharloo et al., 2007).

Estos aspectos subjetivos se reflejan en el modelo mental que las personas con enfermedades crónicas construyen sobre su enfermedad.

Los modelos mentales proceden de los planteamientos de la psicología cognitiva, iniciados por Johnson-Laird (1983) y muestran la tendencia de los individuos de representar el mundo con el cual interactúan. Son producto de una actividad mental constructiva a partir de elementos fragmentarios que el individuo recibe y selecciona de las experiencias vividas, no tratándose de una asimilación pasiva de "copia" de la realidad sino resultado de la percepción, la interacción social y la experiencia personal. Además, no se trata de una representación individual sino que los datos de la experiencia son interpretados y transformados por el sujeto como parte de la representación de la sociedad en la que viven, estableciéndose una dialéctica permanente entre lo individual y lo social. La enfermedad crónica es una parte relevante de esta realidad personal y cultural; pasan a formar parte de la vida cotidiana y como consecuencia, las personas que las padecen generan modelos cognitivos sobre éstas, basados en la experiencia particular y concreta de los síntomas, el conocimiento preexistente y su contexto vital, en el que se incluyen la familia, los profesionales y servicios sanitarios, así como agentes sociales como los medios de comunicación. Estos modelos de enfermedad pueden ser imprecisos, erróneos o inadecuados o bien amplios, técnicos y completos.

La importancia de los modelos mentales radica en que guían la acción, tanto en la adopción de comportamientos, como en la utilización de estrategias de afrontamiento e influyen en las conductas de prevención de salud, estado clínico de la enfermedad (Kaptein et al., 2008), uso de servicios (Frostholm, Fink, Christensen et al., 2005), satisfacción (Frostholm, Fink, Oernboel et al., 2005), reacciones frente a los síntomas, adherencia a las recomendaciones del tratamiento y expectativas de futuro.

Uno de los modelos de enfermedades crónicas más aceptados es el Modelo de Autorregulación de Sentido Común (Common Sense of Self-Regulation Model) propuesto por Leventhal, Meyer y Nerenz (1980) y desarrollado con posterioridad (Cameron y Leventhal, 2003; Hager, 2003).

Este modelo propone que un estímulo (síntoma) genera tanto una representación cognitiva como una representación emocional de la enfermedad. La percepción de la enfermedad sería el resultado de dos procesos paralelos: uno cognitivo y otro emocional, a través del cual todas las informaciones recogidas por múltiples fuentes se sintetizan en una representación de enfermedad que le da un sentido y un significado subjetivo.

Según el modelo de Leventhal et al. (1980), la representación de la enfermedad se organiza en etapas (PachecoHuergo,Viladrich, Pujol-Ribera et al., 2012):

- Cuando se percibe una amenaza para la salud las personas desarrollan paralelamente una representación cogni- tiva de la situación problema y de las emociones que producen.

- Estas representaciones desencadenan planes de acción o afrontamiento como intento de regular la situación.

- Las acciones llevadas a cabo se evalúan (consecución o no de las metas establecidas) y en episodios sucesivos se regula la respuesta mediante un feedback continuo que puede modificar tanto las estrategias de afrontamiento previas como la propia representación de la enfermedad.

Este modelo explica la representación cognitiva de la enfermedad según cinco dimensiones interrelacionadas (identidad, causa, duración, consecuencias y control/curación) también aplicadas a pacientes con EPOC (Calleja, 2013):

- Identidad: esta variable identifica la presencia o ausencia de enfermedad, su denominación y signos y síntomas de la misma.

- Causas: qué conoce el paciente sobre la causa o causas de la enfermedad.

- Duración: expectativa del paciente acerca del tiempo que durará la enfermedad y cómo evolucionará.

- Consecuencias: percepción de las consecuencias de cualquier tipo que tendrá la enfermedad (físicas, emocionales y sociales).

En cuanto a emociones negativas, se ha estudiado la importancia de la ansiedad y depresión en pacientes con EPOC (Jordan, Lee, Valenstein, Pirraglia y Weiss, 2009; Maurer et al., 2008). Se ha encontrado relación directamente proporcional entre percepción negativa de la enfermedad y emociones negativas en los dos sentidos (Hemerle, Olivo, Namerrow y Oz, 2007). Además se han encontrado desesperanza, ansiedad, miedo (a morir, a "quedar sin aliento"), sentimiento de incompetencia y de pérdida de control (Kelly y Lynes, 2008). Se da la circunstancia de que como la expresión emocional favorece la aparición de disnea, algunos pacientes la evitan como mecanismo de adaptación, originando síntomas ansiosos por la represión de emociones. También repercute en la emocionalidad negativa el aislamiento social que produce la limitación de movilidad (Maurer et al., 2008; Medinas, Más y Renom, 2009).

- Control/curación: se refiere a la percepción de la capacidad de la enfermedad de ser curada o modificada por las intervenciones y tratamientos.

De los componentes del modelo de enfermedad que han mostrado tener más capacidad predictiva sobre la CVRS está la dimensión de control, de forma que la sensación de autoeficacia mejora la supervivencia: la enfermedad percibida como modificable y bajo control personal lleva a los individuos enfermos a poner en práctica conductas más saludables, conseguir niveles más altos de adherencia al tratamiento y alcanzar mayor calidad de vida (Kohler, Fish y Greene, 2002). 
La combinación de estos factores dan como resultado la representación de tres modelos de enfermedad (Quiceno y Vinaccia, 2010):

- Modelo de enfermedad aguda: duración corta, causas específicas y consecuencias a corto plazo.

- Modelo de enfermedad crónica: de larga duración, causada por múltiples factores que incluyen hábitos y estilos de vida no saludables y produce consecuencias graves.

- Modelo de enfermedad cíclica: con alternancia de periodos sintomáticos y asintomáticos.

El objetivo de este estudio es conocer el modelo mental que sobre la EPOC tienen los pacientes que la presentan, analizando los componentes del Modelo de Autorregulación de Sentido Común de Leventhal.

\section{Método}

El diseño corresponde a una investigación cualitativa exploratoria, descriptiva, siguiendo una perspectiva fenomenológica.

Los participantes en el estudio forman parte de una investigación previa (estudio ICEPOC (Barnenstein-Fonseca et al., 2011), ensayo clínico aleatorizado con el objetivo de evaluar una intervención multifactorial para la mejora del cumplimiento terapéutico. Los participantes en este ensayo se seleccionan por muestreo no probabilístico, a partir de listados de pacientes con diagnóstico EPOC de cuatro Centros de Salud de Málaga, tres urbanos y uno rural. El nivel socioeconómico es homogéneo entre centros y de carácter medio-bajo, con la particularidad de existencia en uno de ellos (Centro de Salud Trinidad) de dos zonas con necesidades de transformación social (Dirección General de Bienestar Social, 2006), en cuya población concurren situaciones estructurales de marginación social.

Los criterios de inclusión son: diagnóstico de EPOC en su historia clínica; tener prescrito tratamiento inhalado con pauta fija diaria y aceptar participar mediante consentimiento informado. Se aplican los siguientes criterios de exclusión: presentar otros procesos respiratorios no incluidos en la definición de EPOC o padecer problemas físicos o cognitivos (incapacidad para desplazarse, deterioro cognitivo o demencia, déficit sensorial avanzado), que impidan participar en el estudio. Los pacientes con criterios de inclusión se asignan aleatoriamente a un grupo de estudio (se realiza la intervención) y un grupo control (seguimiento clínico habitual). Los participantes en nuestra investigación corresponden al grupo de estudio, formado por 58 pacientes y la recogida de datos se realizó previa a ninguna intervención.

De los pacientes incluidos se recogen: datos sociodemográficos, parámetros objetivos de la enfermedad, tratamiento prescrito y grado de cumplimiento del tratamiento inhalado mediante contador automático de dosis.

La investigación tiene la aprobación del Comité Ético de los Distritos Sanitarios Málaga y Axarquía. La técnica utiliza- da para recoger la información es la entrevista grupal (Mousing y Lomborg, 2012).

Se realizan 22 entrevistas videograbadas entre abril de 2008 y noviembre de 2009 , teniendo lugar en el centro de salud de referencia de cada paciente, previa cita telefónica (hasta tres intentos). El tamaño de los grupos oscila entre 2 y 7 personas con una duración aproximada de 90 minutos. Se utiliza un guión de preguntas que no se formulan directamente, sino que se utiliza como guía para sugerir a los participantes expresarse sobre los temas incluidos.

Análisis: La entrevista grupal una vez videograbada se transcribe literalmente, siguiendo los criterios de Jefferson (1985). Tras la lectura y relectura de la transcripción, se segmentan los párrafos según su relevancia temática y se asignan etiquetas verbales siguiendo una estrategia inductiva. Para la codificación se utiliza el programa Atlas.Ti 5.2. Se revisa el listado inicial de códigos eliminando errores y fundiendo códigos redundantes. Se utiliza el diccionario de la Real Academia de la Lengua para valoración semántica de términos. Se introducen códigos nuevos hasta obtener la saturación, definida por el momento en que se utilizan exclusivamente códigos preexistentes ("by list"). Se sigue el método comparativo constante entre los distintos elementos de análisis (grupo-grupo, código-código, investigadora-investigadora). Los códigos se organizan en familias y se relacionan formando redes y mediante operadores booleanos y semánticos hasta obtener las categorías centrales y sus relaciones.

Todo el análisis se realizó por dos investigadoras trabajando por separado la codificación y consensuando las categorías y redes.

Los procedimientos de rigor metodológico utilizados se resumen en la tabla 1 , incluyendo la verificación del informe provisional por los propios participantes, realizándose 3 sesiones adicionales para la presentación-discusión, siguiendo el mismo formato de entrevista grupal videograbada. Las aportaciones realizadas se incluyen en el informe final.

Tabla 1. Procedimientos de rigor metodológico.

\begin{tabular}{ll}
\hline Credibilidad & Devolver a los pacientes participantes los \\
& resultados del estudio para su validación. \\
\hline Conformabilidad & Recogida precisa de todos los pasos reali- \\
& zados durante la investigación, a fin de po- \\
& der ser reproducidos. \\
- & Recogida de datos por parte de investiga- \\
& dores no implicados en seguimiento clínico \\
& de los pacientes. \\
- & Realización del análisis por investigadores \\
& no implicados en seguimiento clínico ni en \\
& recogida de datos. \\
- & Contraste entre investigadores. \\
\hline Transferibilidad & Recogida de datos contextuales suficientes \\
& y de calidad. \\
- & Criterios de inclusión precisos. \\
\hline &
\end{tabular}




\section{Resultados}

El perfil del participante (Tabla 2) corresponde a un hombre de 68 años de edad de bajo nivel cultural, con diagnóstico de EPOC de unos cinco años de evolución, con un nivel de gravedad medio y exfumador. Tiene pautado tratamiento inhalado y frecuentemente no sigue correctamente la pauta de tratamiento.

Tabla 2. Perfil del paciente participante en el estudio ( $\mathrm{n}=58$ ).

\begin{tabular}{ll}
\hline VARIABLE & RESULTADO \\
\hline Sexo & Varón $(91.4 \%)$ \\
Edad & $68.59 \pm 7.68$ años \\
IMC & $30.48 \pm 4.81 \mathrm{~kg} / \mathrm{m}^{2}$ \\
Nivel de estudios & Primarios o inferior $(72.4 \%)$ \\
Relación con el tabaco & Exfumador $(63.8 \%)$ \\
FEV1\% medio & 70.6 \\
FVC $\%$ medio & 84.11 \\
FEV1/FVC medio & 61.46 \\
Gravedad EPOC & Leve $(39.7 \%)$ \\
& Moderada $(37.9 \%)$ \\
Fenotipo & No exacerbador (menos de 2 \\
& reagudizaciones al año) $75.9 \%$
\end{tabular}

Cumplimiento tratamiento inhalado* $41.4 \%$

Nota IMC: Índice de Masa Corporal. FEV1: volumen espiratorio en el primer segundo de espiración forzada FVC: capacidad vital forzada EPOC: Enfermedad Pulmonar Obstructiva Crónica.

* 80-110\% dosis pautada mediante contador automático

Analizamos la visión de la enfermedad dada por los pacientes en las cinco dimensiones del Modelo de Autorregulación de Sentido Común de Leventhal (Tabla 3).

Identidad de la enfermedad: existe confusión en los pacientes sobre la denominación de la enfermedad como EPOC y tienen dificultad para diferenciarla de otras enfermedades.

El diagnóstico se realiza en fase avanzada de la enfermedad, siendo confusa la forma de llegar a éste:

1) En reagudizaciones de la EPOC no diagnosticada previamente ("resfriados" frecuentes, reagudizaciones severas): P.2.2:...antes yo me resfriaba, se me cogía a los bronquios,...ya di con este médico que me dijo que habia que buscar el origen...y empezó a hacerme pruebas. También por presencia de otras enfermedades respiratorias no relacionadas (neumotórax, tromboembolismo, apnea del sueño).

2) En presencia de problemas no respiratorios, fundamentalmente cardíacos, con los que comparte el síntoma disnea, y también en presencia de hechos fortuitos como accidentes con traumatismo torácico.

Como técnicas diagnósticas se mencionan la radiografía y la espirometría. Se reconoce a la espirometría (nombrada de distintas formas: "prueba de soplar", "aspiraciones") como técnica de medida de la capacidad respiratoria (P3.3: ..me dieron un pase para eso de "soplar".. y me dijo, mire usted yo lo que veo aqui es una persona de setenta y dos años que ha sido fumador y entonces tenía un cincuenta por ciento de capacidad), así como para el seguimiento, con capacidad de indicar mejoría o empeora- miento: P5.1:me hizo una prueba de aspiraciones y dijo que estaba bastante mejor. P8.1: me hacía la prueba de soplar y cada vezpeor.

Tabla 3. Principales confusiones manifestadas por los participantes.

\section{ASPECTO TEXTO EJEMPLO}

Identidad P9.3: $y$ no se ni lo que tengo.P11.3: algo de bronquios $y$ bronde la quitis también.P22.1:Yo en principio es que me acabo de enteenfermedad rar de lo de EPOC....lo de que tenía EPOC me he enterado porque ustedes me han llamado. Vamos que no lo sabia,...cuando tengo asi un ataque de bronquios, bronquitis, catarro asi fuerte cogido,....pero lo de EPOC...

Causas TABACO: P7.1: Con el tabaco nos estamos quitando la vida a diario. OTRAS:P21.4: Mi trabajo era de madrugar mucho, me he resfriado mucho y me ha atacado a los bronquios. P6.4: Yo con los bronquios llevo mucho padeciendo, yo me dedico a la construcción y el polvo me ha hecho polvo, el polvo es muy malo para los bronquios; P16.2: pero yo creo que esta enfermedad tiene también un componente hereditario, porque mi abuelo recuerdo yo que tenía este problema.

Papel de los CAUSA: P9.2: Claro, un resfriado mal curado, eso lo tengo "resfriados" yo crónico de toda la vida.

EMPEORAMIENTO:P.20.1: Y un resfriado que me mata. P2.2:..cuando me resfrío, yo me resfrío y ya se me viene todo encima.

TIPOS, ACTITUD TERAPEÚTICA: P4.3: $i Y s i$ es un resfriado digamos común? Que es el que casi siempre tengo, entonces no me lo tomo, no es un resfriado de gripe ni mucho menos, entonces no me lo tomo, que tampoco quiero abusar mucho de los antibióticos, él me dijo "en cuanto te resfries te lo tomas" pero claro, pienso que cuando me resfríe de otra manera.

VULNERABILIDAD: P12.3: Yo creo que, al tener de bronquios pues, si no lo tienes a lo mejor es más pasajero, o lo que sea, creo yo.P12.3: y al estar tocado de pecho apretará más o no lo se...

CUIDARSE=EVITARLOS: $P 12.2: \quad y \ldots$ trato de no... y de no ponerme donde pueda yo coger frío, cosas que me puedan causar un constipado, porque si me constipo estoy peor. En fin, trato de cuidarme, y a veces pues... sí, parece que lo consigo

Evolución CRONICIDAD, PROGRESIÓN: P13.3: y es cada día peor, claro. Pero que esto no tiene ya arreglo. Que cura no tengo ya.P6.2. Yo creo que es para toda la vida por mucho que uno haga creo que sí, que eso va a ser para la eternidad.

ALTIBAJOS: P2.1: esto es crónico, épocas mejores, también el frío que está haciendo este año, ..... lo notamos mucho, yo por lo menos lo noto. P14.2: cuando llegan los inviernos, me resfrí mucho, me pongo fatal, y...y eso.

MEJORÍA: P12.2: Y bueno, pasito a pasito, si se cuida, esto va, ahora, como no se cuide esto no va muy lejos. P12.2: Ahora, eso sí, hay que cuidarse mucho, si me cuido, vas a mejor;

EFECTO DE LA EDAD:.P1.2: Bueno como vamos para mayores, esto es una lotería que nos ha tocado...

Síntomas PLURIPATOLOGÍA: P2.1: me falta el aire pero por el calor que me da el azúcar, pero no por la EPOC.

INFRAVALORACIÓN: P11.2: ..para mí yo respiro bien, normal, cuando no estoy resfriado, cuando no corro mucho. Cuando ando despacito no me pasa nada. Abi mismo respiro yo bien... P13.1: Todavía cuando subo, o ando por el 


\begin{tabular}{l}
\hline $\begin{array}{l}\text { ASPECTO } \\
\text { CONFUSO }\end{array}$ \\
$\begin{array}{l}\text { campo y tengo que subir las cuestas, o me canso mucho, pues } \\
\text { me da una "mijilla" de asfixia, pero no lo noto yo, normal- } \\
\text { mente no me suele dar. }\end{array}$
\end{tabular}

Diagnóstico POR REAGUDIZACIONES:P12.2: venía bastante mal,...y vine a la urgencia, me dice, esto es EPOC. OTRAS ENFERMEDADES RESPIRATORIAS: P12.1: ... alli en el hospital, una trombosis de pulmón que me ha dejado secuelas, y entonces, a raí de eso..., P 1.1: ..también tenía apnea del sueño, entonces me bicieron pruebas y ya me sacaron La EPOC P13.1: porque me dio un neumotórax espontáneo, ...y y a empezaron a salir a relucir...pues tenía EPOC...

\section{OTRAS ENFERMEDADES NO RESPIRA-}

TORIAS: P18.1: Esto ocurrió porque tuve una arritmia P3.2: me di cuenta a raíz de un accidente de coche, tenia mucha presión, no podía respirar bien..

Creencias P8.1: ... y me mandaba aerosoles y mi hija "no te lo pongas erróneas mamá, que eso no es bueno".P12.4: Esa medicación cuando te sobre inha- la ponen, es para siempre.P5.2: Yo lo tomo una vez por la ladores mañana y para no acostumbrarme, cuando estoy muy fatigoso por la noche... pero yo nada más quisiera tomarlo por la mañana para no acostumbrar mucho al cuerpo...

P13.2: Yo lo único que quiero es usar menos de esto...y si puedo, mientras menos mejor.

Pautas de P17.2: abora me lo tomo cuando veo que lo necesito, cuando tratamiento no, no. y la época del año.

P13.2: ...esto lo tengo provisional, como aquel que dice, para el caso de una asfixia.P6.3: ...el otro tiene unas horas, y el otro es para cuando sienta la fatiga.P6.4: Porque si tú estás muy resfriado, tú mismo te indicas, pero si estás un poquillo mejor, pues los bronquios están mejor.
La broncoscopia es recordada como muy desagradable (P2.2:...no lo volveré más en la vida, como sería que dos que habia para entrar dicen. "¿usted ha sido el que...jufi nos vamos;". Aquello era horroroso, vamos...pasé lo que no está en los escritos, decía "es molesto",..., molesto...no lo volveré más en la vida).

Respecto a las causas (Figura 1), se considera a la enfermedad fundamentalmente debida al tabaco (P3.2: Para mi un exceso de fumar, fumar mucho. Yo fumaba muchísimo era viajante y mi compañero era el tabaco y yo fumaba muchísimo) y se conoce la forma en que el tabaco produce la obstrucción al flujo aéreo (P21.2: Yo para mi fue el tabaco que te va cerrando, te va cerrando y cuando quieres darte cuenta el alquitrán ya se habrá enquistado, se habrá aposentado y entonces ya tienes que usar el inhalador para poder respirar mejor).

Son muy relevantes para los pacientes otras causas como son las infecciones respiratorias (P6.3: De los resfriados; P7.2: Yo tengo los bronquios de los resfriados mal curados;), exposición a humos y polvos, trabajos duros desde la juventud y factores hereditarios (Tabla 3). Se manifiestan dudas del papel real y exclusivo del tabaco, admitiéndolo con afirmaciones vagas y mostrando que la atribución de la enfermedad al tabaco procede más de los profesionales de la salud que de la convicción propia (P13.2: pues yo::: los médicos me lo echan al tabaco) y en general, se cree que "tiene que haber algo más". Así, la posición ante el tabaquismo es confusa (Tabla 4): aunque se señala como causa fundamental y factor determinante de la cronicidad, algunos pacientes perciben que no les perjudica e incluso que empeoran al dejar de fumar.

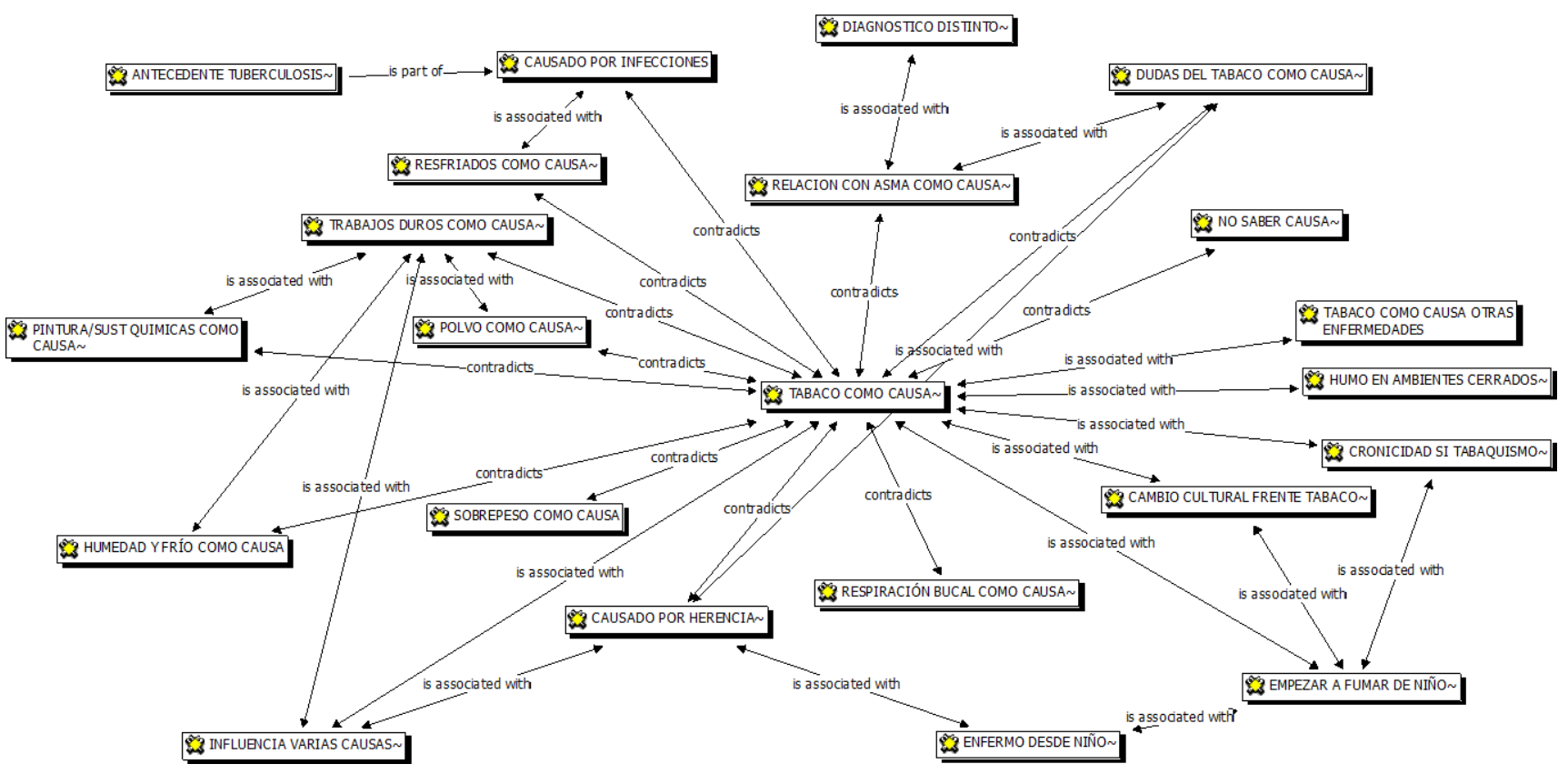

Figura 1. Red de relaciones sobre causas de la enfermedad. Is associated with: está asociado con; contradicts: contradice 
Tabla 4. Ejemplos de narraciones acerca del tabaquismo.

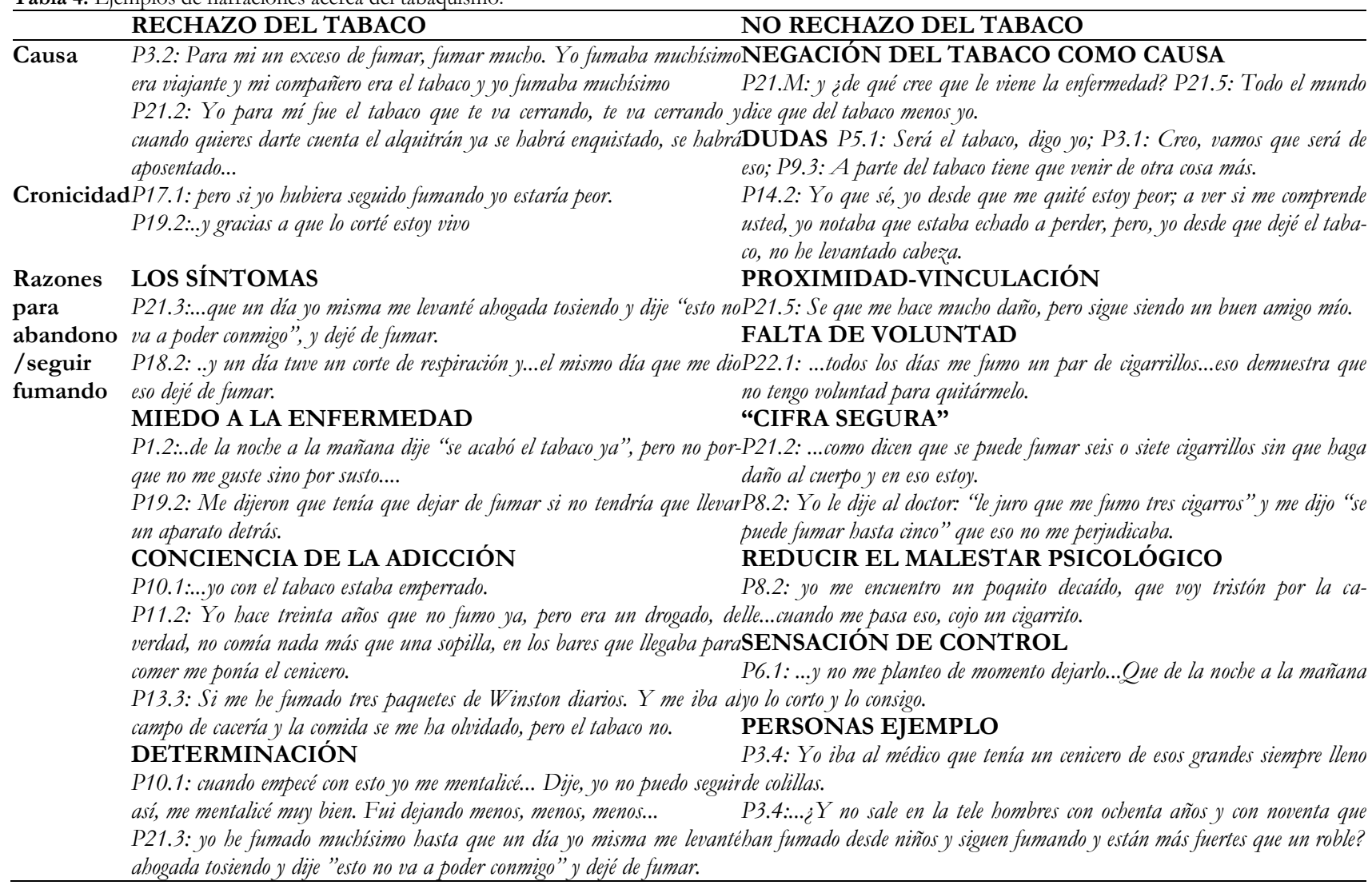

Entre las razones para dejar de fumar están los síntomas, el miedo a la enfermedad y tomar conciencia de la adicción. La clave fundamental parece ser la "mentalización", reconociéndose el importante esfuerzo que cuesta dejarlo (P13.2. Trabajillo me ha costado. P19.2:..bueno, me costó muchísimo trabajo, yo era fumador, fumador, me costó mucho trabajo dejarlo)

Los exfumadores se muestran incluso "duros" con los que no lo dejan: P1.2: A mi me molesta escuchar a la gente decir "es que no puedo quitarme del tabaco, no puedo"; no puedes no, no quieres.

Los pacientes que siguen fumando utilizan diversos argumentos: vínculo, falta de voluntad, percepción de fumar una "cifra segura", reducir el malestar psicológico, sensación de control e imitación de ejemplos como profesionales de la medicina que fuman o personas muy fumadoras sin daños por el tabaco. El paciente que sigue fumando muestra ambivalencia y se justifica en "fumar poco": P9.3: Yo si fumo pero no mucho, lo menos posible.

Se reconoce la existencia de un cambio cultural frente al tabaco en los siguientes aspectos:

- Respecto a la percepción del daño provocado en uno mismo y en los demás (P2.3: 3: Ya ves, la ignorancia que teníamos, que aquella salita mía parecía Londres un día de niebla y mis niñas que eran pequeñas alli también y tanto ellas como yo también bemos respirado eso, porque ahora tenemos otra cultura y sabemos...).
Respecto al inicio precoz del consumo alentado en el entorno familiar (P14.1: Y es lo único a lo que me enseñó mi abuelo, a fumar, con ocho o nueve años).

Respecto al aumento del consumo en las mujeres (P3.3: Las fumadoras ahora están, vamos de años atrás han empezado ahora a fumar y el hombre lleva desde los tiempos remotos y las mujeres desde los sesenta y esos jaleos).

Se presenta gran confusión respecto a los resfriados, con un papel crucial de éstos en toda la enfermedad: como causa (P19.2: de los resfriados mal curados), motivo de empeoramiento e indicador de cambio en la pauta de tratamiento. Se distinguen entre resfriados comunes y "fuertes" (P6.3: Primero empieza el resfriado nasal después se me pasa a la garganta y luego se me agarra al pecho). "Cuidarse" es básicamente evitar los resfriados (P12.2: Ahora, eso sí, hay que cuidarse mucho en el aspecto ese, ¿eh? Porque el resfriado hay que::::) por encima de otras conductas beneficiosas que no se mencionan, como dejar de fumar o cumplir con el tratamiento prescrito.

Duración: Se indica un patrón evolutivo marcado por la cronicidad y el empeoramiento irremediable (P17.2: ... del alquitrán te mueres de viejo y no se te quita...porque lo tienes pegado (se señala el pecho) pegado a los pulmones. Y lo tengo). Dentro de la cronicidad se aceptan distintas maneras de evolucionar la enfermedad, con altibajos (determinados por cambios estacionales o por resfriados) o progresivamente a peor. 
La evolución de la enfermedad es confusa porque se mezcla con el envejecimiento natural, con un ajuste gradual a las limitaciones y con un efecto de "acostumbramiento". El efecto de la edad se confunde con la evolución de los síntomas, existiendo tendencia a compararse con la juventud y asociando la progresión al envejecimiento (P17.4: Yo creo que vamos a empeorar según la edad. Casi todos morimos de una enfermedad pulmonar y ya está).

Consecuencias de la enfermedad: la presencia de la enfermedad se manifiesta sobre todo por los síntomas (Tabla 5), existiendo una tendencia general a su infravaloración (P1.1: llego un poco cansado pero eso es normal.).

Los pacientes con peor percepción de su estado muestran una visión global de gran deterioro de su salud, sin referencia a síntomas concretos (P5.2: pero malo, yo estoy muy malo. P5.2 Yo estoy fatal muy mal.).

Los síntomas más relevantes para los pacientes son los más "visibles", utilizándose metáforas muy sensoriales: la "sinfónica" (sibilantes), el "chaleco" o "corpiño" de dolor, la "chimenea" (sensación de ocupación de la vía respiratoria) o la "colmena" o "lapa" (moco en la vía respiratoria). La tos es un síntoma muy molesto y en ocasiones bastante intenso. Los "pitos" son un síntoma indicativo de empeoramiento (reagudizaciones) por resfriados y resulta un alivio hacerlos desaparecer. Es importante la sensación de obstrucción por los "mocos" como responsables de no poder respirar bien, y lo que se pide al tratamiento es "desatascar", "arrancar", "diluir" o "expulsar" la mucosidad.

El síntoma asfixia ocasiona importantes repercusiones. Es señalado como motivo clave para dejar de fumar y se considera indicador de gravedad y de progresión de la enfermedad y a la vez es un marcador de mejoría por el uso de tratamiento.

Aparecen en las entrevistas síntomas no respiratorios como el cansancio y el dolor. El cansancio se refiere a agotamiento, falta de capacidad funcional y en algunos casos parece confundirse con la disnea. El dolor en el pecho es también un síntoma frecuentemente señalado, a veces extendido al resto del cuerpo.

Respecto a las reagudizaciones, también suponen un aspecto de confusión. No se las identifica como tales (a la manera de "brotes" en otras enfermedades crónicas) sino como "resfriados" o "bronquitis" (episodios aislados no claramente relacionados con una enfermedad de base) (P22.1: cuando tengo así, un ataque de bronquios, bronquitis, catarro asi fuerte cogido, que me da por toser por las noches y eso). En ocasiones son muy fuertes e incapacitantes (P7.2: 2: yo lo único es que cuando me resfrío me tengo que meter en la cama con cuatro o cinco cajas de antibióticos).

El temor a las reagudizaciones se manifiesta por los pacientes como miedo a los resfriados (P22.1: Estoy un poco acobardado, sí, por si me resfrío y con miedo a que se me repita, se señala el pecho; P11.3: pero le temo a un resfriado porque eso es para mi una cosa mala; P11.3: pero eso, temiéndole mucho a los resfriados) y lleva a autoimposición de limitaciones (P8.2: a mi me preocupa el res- friarme, y a veces no salgo a ningún lado por no coger frío por los cambios bruscos de temperatura).

Tabla 5. Síntomas de la enfermedad.

\section{SÍNTOMA TEXTOS EJEMPLO}

P10.1: me daba una tos que me quedaba traspuesto, que me arecía que me iba de tanto toser.

Tos P13.3: me entraba la tos, una tos continua, pero muy fina, tacatacata y de buenas a primeras ipom; caía.

P11.3: Si me da la tos, eso no se lo recomiendo a nadie. Es una tos continua, las veinticuatro horas del día.

P17.1: yo por la noche, me asfixiaba, mi mujer me pegaba porrazos en la espalda porque es que me faltaba el aire, me faltaba la vida.

Asfixia

P9.4: y ahora llevo tres años yendo al medico y tengo enfisema pulmonar severo y me asfixio muchísimo

P1.2:...apenas me resfriaba empezaba "la sinfónica" a funcionar y ahora, a Dios gracias, ahora los pitos han pasado a la bistoria.

P7.3: .. a mí lo único que me impide respirar bien es la mucosidad.

P4.3: tengo mucha mucosidad, yo no se de donde me sale a mí tanta mucosidad.

P8.2: una mucosidad de hebra blanca pegajosa como las lapas que se pega a la garganta y a los bronquios.

P3.3: lo mismo que las colmenas está pegado abi el alquitrán ese.

P4.3: los jarabillos esos me he tomado todos los habidos y por haber y hombre, me lo ablanda, pero yo me imagino que no termina de romper la mucosidad que haya abi apostada.

Dolor P.2.2: me tenía que agarrar cuando tosía porque era a puro dolor, yo decía, tengo un corpiño de dolor, asi como si fuera un chaleco, asi era el dolor cuando tosía.

P12.1: Yo estoy que me duelen las piernas, me duele el cuerpo, me duele todo.

Cansancio P13.3: yo no lo sé si entra poco aire o estoy cansado, sentado estoy cansado.

P21.4:..yo me canso mucho, me agoto mucho, la escalera no puedo subirla.

P4.2....el inconveniente es que me canso mucho y me asfixio

ADAPTA- dormir sentado o incorporado:

CIONES: P16.2: Cuando tienes una crisis que estás peor, si es de noche, procuras incorporarte...Si o dormir un poco incorporado.

P16.3: Sentado, en vez de acostado, sentado,

pararse mientras va caminando:

P14.1 A mi me cuesta subir las cuestas, abi es donde me asfixio, una cuesta arriba, andando normal yo, o me paro o me asfixio.

P13.1: Para mí, cuando esfuerzo el cuerpo más de lo normal me siento una mijilla, me siento unos minutillos $u$ después sigo,

"agarrarse" a sitios de la calle:

P20.1: El truco es que si voy por la calle, agarrarme a un árllo a una ventana, espero un rato y luego echo a andar.

abrir la ventana:

P21.2: Yo me abro la ventana, ya puede ser invierno, yo abro la ventana una hoja abora en invierno...

P17.2....abría mi ventana de par en par

Es frecuente el cambio de la pauta inhalatoria durante la reagudización (P2.2: ...cuando estoy resfriado me hago una por la 
mañana, otra al medio día y otra por la noche dos veces, y luego el otro que es asi, dos veces también).

Respecto a las consecuencias de la enfermedad en la calidad de vida, en especial se señalan la afectación del sueño y la limitación de la movilidad. Las limitaciones se hacen extensivas a la familia: P2.1: ...yo antes acompañaba a mi mujer que viajábamos con esto del INSERSO, y te obliga a dejar de viajar, pero en fin, hay que reconocer las limitaciones, es muy molesto, porque a veces estás en la calle y te notas que te falta el aire, y te tienes que ir a la casa, total, que estoy limitado y tengo limitada a ella por vínculo matrimonial...

Los sentimientos asociados a la EPOC son sentimientos del tipo desesperanza, resignación (P1.2:... esto es una lotería que nos ha tocado y tenemos que sobrellevarla; P14.2: y asi que...esa es la carga que llevo) e indefensión (P3.2: Yo lo que si veo es que a mi me tendría que desaparecer, que no me desaparece esa asfixia, ¿Por qué a mi no me desaparece? Si yo no fumo...).

También aparecen sentimientos de miedo, asociados a la progresión de la enfermedad y a verse dependientes en el futuro. Necesitar oxígeno llega a causar más impacto que el miedo al cáncer (P1.1: uno escucha que si el cáncer, que si esto, que si lo otro, pero una noche salió en la tele un informativo con esa enfermedad y los señores tenían que ir con bombonas de oxigeno y eso le da a uno por pensar).

Existe también miedo a los efectos del tabaco, que son relevantes más en la experiencia ajena que propia (importancia del aprendizaje vicario) (P1.2:.. de la noche a la mañana dije "se acabó el tabaco, pero no porque no me guste, sino por susto, porque un tío mío se me murió en los brazos con un edema pulmonar..).

Así mismo, los participantes manifiestan temor al uso de inhaladores (P13.2: Yo lo único que quiero es usar menos de esto...y si puedo, mientras menos mejor.) y a hacerse adicto o dependiente de la medicación inhalada (P5.2: pero yo nada mas quisiera tomarlo por la mañana para no acostumbrar mucho al cuerpo ¿no?).

Control/curación de la enfermedad: existe importante confusión en los pacientes acerca de su capacidad de intervenir sobre la enfermedad. La visión general se inclina al pesimismo, asumiendo un empeoramiento inmodificable: P6.2. Yo creo que es para toda la vida por mucho que uno haga creo que sí, que eso va a ser para la eternidad.

A pesar de la cronicidad, se plantea la posibilidad de estabilizar la enfermedad mediante hábitos de vida sanos: M.21: ¿Y cree que esto va a ser para toda la vida?, P21.3: Yo pienso que no. Yo pienso que abora que he empezado el régimen y cuando yo adelgace un poquito y haga una vida más sana y empiece a andar, que me he ido ahora a vivir al Rincón que estoy entre campo y playa, en una zona de mucha naturaleza.

Entre los hábitos de vida que valoran como beneficiosos destacan el ejercicio físico (P9.4: Yo me voy a andar, si eso es buenísimo, hay que andar); la toma de abundante agua; bañarse en el mar (P2.3: A mi me gusta mucho irme a la playa a bañarme....Y Ya ves, el mar, estupendamente...eso para los bronquios es buenisimo, el agua del mar..).; la respiración profunda (P5.1: ...cuando me noto un poquillo..me paro, empiezo a hacer la respiración, a respirar hondo, a aguantar un poco y se me va pasando) y respirar aire puro, del campo (P13.1: el aire es más limpio en el campo que en la ciudad y te encuentras mejor). Se mencionan como beneficiosos la balneoterapia y la fisioterapia respiratoria (P9.4: Yo estuve también en terapia respiratoria eso si que se lo recomiendo yo a todo el mundo eso es estupendo). Resultan adaptativos mantener buen humor y las aficiones: P10.1: Yo a pesar de que tengo lo que tengo, pero tengo muy buen humor. $Y$ toco la guitarra también, flamenca....

Respecto al tratamiento farmacológico existe importante confusión en los pacientes (Tabla 6). Las expectativas de efecto del tratamiento se centran en "limpiar" el material que obstruye los bronquios (P18.1: ...ojalá sacaran algo que te dejara limpio de pronto.:). El tratamiento inhalado no se reconoce como "medicación" (P19.1: Yo no estoy tomando nada. M2: ¿No tiene ningún aerosol?.P19.1: No, tengo una "polvera" de esas").

En cuanto a los efectos teóricos de los inhaladores, muchos pacientes los reconocen como broncodilatadores, aunque se mencionan otras propiedades como las antiinflamatorias y en el sentido de "limpiar". Respecto a los efectos reales, la mejoría, si existe, es reconocida de distintas formas: como no resfriarse, como tener menos síntomas (pitos, disnea, mocos, dolor), como una mayor capacidad de realizar actividades y como menor duración de la reagudización (P22.1: Si, yo he notado que tengo alivio con los inbaladores,...pero vamos, que cada vez. tardo más en que se me cure, ahora me dura casi un mes, antes me duraba una semana el ataque de bronquitis asi de fuerte, me dura un mes seguido y no estoy bien del todo).

Aparecen muchas dudas sobre la efectividad de los inhaladores y muchos pacientes manifiestan no notar beneficio alguno, ni diferencia entre usar o no el inhalador.

Es importante el tiempo que pasa entre inhalar y notar el efecto:

P17.2: Pero el alivio lo siento, pues a los dos minutos siento el alivio.

P21.5: hombre, no es una cosa inmediata, pero luego sí.

P11.3: tarda a lo mejor un tiempo, pero se quita.

P3.3: No es como si te duele la cabeza y siempre, de toda la vida, ha dicho uno, "tómate una aspirina". Yo tomaba la aspirina y al rato tú decías, "oye, es verdad que se me ha quitado el dolor". Esto no, esto a lo mejor te alivia, pero no es una mejoría de decir...

Respecto a cuánto debe durar tratamiento inhalado, aunque lo reconocen como permanente (P19.2:... a mi me mandaron este tratamiento para toda la vida. P18.1: yo no lo abandono desde luego, es que es para toda la vida), existe una tendencia general a usar los inhaladores cuando "se está resfriado": P6.4: ..yo me lo estoy tomando ahora porque he estado muy resfriada. P13.2: ...esto lo tengo provisional, como aquel que dice, para el caso de una asfixia.

Los efectos secundarios reconocidos son lesiones bucales, taquicardia, nerviosismo, irritabilidad y agresividad.

Respecto a la técnica inhalatoria, dan mucha importancia a "notar" o "no notar" sensaciones físicas cuando realizan la inhalación (P12.2:..en qué se notará, en qué se notará que eso sea bueno o sea malo, yo no sé, porque yo no sé, eso es tomártela y ya está, porque yo no sé, eso no sabe a nada). Esta importancia de los aspectos físicos, se manifiesta en la descripción de los inhaladores por sus colores, formas y sonidos...P3.4....y me dijo, te voy a mandar otra que es como una galleta. P5.1: lo que el de rosquita 
ese, me parece a mi que no lo hago bien, el jclick; jclick;. P9.2: Y el que más noto es el blanco y el azul.

En general, conocen la técnica de la inhalación y las normas de uso aunque reconocen que les costó aprenderlo (P3.2: ...lo que si es verdad es que me ha costado trabajo aprender a echármelo para que llegue a los pulmones).

Tabla 6. Ejemplos de manifestaciones acerca del tratamiento.

EXPECTATIVAS P3.2: lo que si es verdad es que tendría que inventar un medicamento que diluyera eso que nos presiona los bronquios .... ¿no quitan la obesidad, quitan la grasa? Pues ¿̇por qué... a los bronquios no le pueden quitar eso que es molestísimo? El no poder respirar es fatal. P13.2: que ojalá sacaran algo, ....que limpie más, que limpie más, P3.2: a mi lo que gustaría encontrar un medicamento que fuera diluyendo el alquitrán...

P3.2: a mi me hace falta un deshollinador, pum pum, que me limpie ese algo que...

EFECTOS BRONCODILATACION P9.2: Para que llegue un poquito más de oxígeno, te dilata.

(teóricos) P6.1: Yo sé que son broncodilatadores ¿no? Entonces se entiende que dilataran los bronquios para que haya más espacio para que el aire salga.

ANTIINFLAMATORIO P16.2: Pues será como un antiinflamatorio, para evitar la inflamación de los bronquios.

LIMPIEZA P3.3: Será como un detergente que va limpiando para que entre aire en los pulmones.

EFECTOS NO RESFRIARSE P1.2:..yo me siento bastante mejor que antes, prueba de ello es que antes cada dos por tres estaba resfriado y (percibidos) en mi casa somos seis, todos han caído resfriados y yo todavía me mantengo.

CONTROL DE SÍNTOMAS P1.2: yo antes de tomarla apenas me resfriaba empezaba la sinfónica a funcionar, y abora, a Dios gracias, ahora los pitos han pasado a la historia.P11.2:Yo lo que esputo mucho. Y eso me quita la carraspera, hago "click" $y$ limpio.

AUMENTO DE CAPACIDAD DE REALIZAR ACTIVIDADES P1.1: Yo ahora, incluso subir escaleras,....y nada de abogos. P3.3: yo encuentro mejoría, yo no podía ni agacharme para los cordones de los zapatos. P21.3: Eso si lo he notado yo, que aspiro más aire, a mí me gusta mucho cantar, yo siempre estoy cantando en mi casa y antes como no me llegaba y abora si cojo aire, me Llega.

BENEFICIO POR DEFECTO P3.2: Yo sé que si no me lo tomo, yo me lo noto... P8.2: ...pero cuando no me hago los aerosoles, caigo de momento.

P21.5:.. y la prueba mía es dejar de tomarla, la prueba para saber si me sirve.

EFECTO DUDOSO P3.3: Sí, vamos, a mi caerme mal no me puede caer, ahora, que haga más o menos.

P12.2: ahora, el efecto que hace ahi dentro, o lo que pasa, yo no sé por dónde sale.....yo que sé dónde irá eso...pues irá....algo hará.

EFECTO NULO P6.1: No noto nada...echarme el spray y después estoy igual, yo no noto nada...

P16.1: Yo es que no noto diferencia si me la tomo o no me la tomo. Entonces, no sé.

P14.2:...yo ahora, a mi es que no me sirve para nada.

EFECTO DISTINTO AL ESPERADO. P8.1....llevo ocho años tratándome la enfermedad y yo no encuentro que esa mucosidad blanca pegajosa se me quite... P13.3: ... yo lo que veo que, que hará algo, pero no lo que uno quiere.

EFECTOS BOCA P12.1:.. y a mi me afecta normalmente la boca y yo a los inhaladores esos se lo achaco que be perdido yo toda la dentadura, y INDESEABLES pellejos en el cielo de la boca..

NERVIOSISMO P17.2: ..el aerosol me da muchos nervios. ..me atacan los nervios...eso es lo que más malamente me cae a mí. P21.2: es que eso me pone tan nervioso que soy capaz de darle una torta a uno sin motivo.

TÉCNICA P4.2: El polvillo ese que suelta lo noto entrar de la campanilla para abajo.P12.1: Yo lo que noto es un sabor que te queda en la boca, un sabor...P17.3: ...eso se nota enseguida, porque no, porque no vibra la cápsula.

P18.1: Y hace ruidito la pastilla cuando la preparas P5.1: lo que el de rosquita ese, me parece a mi que no lo bago bien, el jclick; iclick;.

Existen creencias erróneas respecto a los inhaladores: son "malos" (P8.1: .... me mandaba aerosoles y mi hija "no te lo pongas mamá, que eso no es bueno..);:cuando se inician ya no se pueden dejar (P12.4: Esa medicación cuando te la ponen, es para siempre) y el ya mencionado "acostumbramiento".

Las pautas de tratamiento son bastante confusas para los pacientes. En unos casos manifiestan seguir continuada y correctamente el tratamiento (P14.1: Yo la sigo tomando, para los bronquios, me da igual estar bien que mal, me lo tomo todos los días..), mayoritariamente las dosis cambian según el estado del paciente y la época del año, bien a iniciativa propia o bien por indicación médica.

El cumplimiento lo relacionan con notar el beneficio (P10.1: ..estoy mejor, pero no lo dejo, porque me va bien; P17.4: Es que yo, yo no puedo dejarla ya. El estado que yo tengo ya de asfixia...lo que pasa es que como la deje jcatapúmi).
Aparte la medicación inhalada, se habla del efecto de los expectorantes, más "cercanos" al efecto esperado (P4.3: ...cuando tomo jarabe noto que me expectora, noto como si se me desatascara eso; P4.3:...lo que pasa que ya los jarabillos esos me he tomado todos los habidos y por haber y, hombre, me lo ablanda, pero yo me imagino que no termina de romper toda la mucosidad que haya abi apostada).

Consideran los antibióticos necesarios pero con efectos adversos sobre el estado general: P11.3: Resulta que los antibióticos me van mal, me quitan de comer, se me quita todo, me entra una crisis de debilidad que me dura un mes o dos meses.

También es confusa la acción de la vacuna antigripal, que tiene adeptos (P7.3: yo antes no me vacunaba pero ya llevo dos años vacunándome y desde entonces no be vuelto a coger un resfriado; P19.2: y me vacuno todos los años, me va muy bien) y detractores (P8.2: ...en mala hora me vacuné, en mala hora. Desde que me puse la vacuna mi cuerpo parece un pozo de petróleo, a ver si usted me entiende). 


\section{Discusión}

El resultado principal de nuestro estudio es la falta de un modelo mental establecido sobre la EPOC en los pacientes que la padecen, encontrándose una alta confusión en los diferentes componentes de la representación de la enfermedad según el Modelo de Autorregulación de Leventhal: respecto a la identidad, la enfermedad tiene poco reconocimiento como tal, se diagnostica en descompensaciones y se confunde con otras enfermedades; la causa se atribuye no sólo al tabaco sino a "algo más" (humos, resfriados, trabajos duros); la duración se identifica como crónica, con altibajos y se confunden exacerbaciones y "resfriados"; en cuanto a las consecuencias, se minimizan los síntomas, y se mezclan con los de otros trastornos; no se tiene percepción de poder ejercer control sobre la enfermedad, con confusión de las pautas de tratamiento y de los efectos de la medicación inhalada, existiendo creencias erróneas sobre ésta.

El perfil del participante en nuestro estudio es similar al encontrado por De Miguel et al. (2004): hombres, edades medias-altas, pensionistas, bajo nivel educativo, aunque no se correspondería con el obtenido en grandes estudios poblacionales (Miravitlles et al., 2009): menor nivel cultural (estudios primarios o inferior $72.4 \%$ ) y mayor gravedad (solo $39.7 \%$ leves) en nuestros pacientes.

Encontramos importantes dificultades para extraer de las narraciones una representación cognitiva de la EPOC.

Siguiendo los componentes del Modelo de Autorregulación de Sentido Común (Leventhal et al., 1980), respecto a la identidad, se aprecia una falta de conocimiento sobre el nombre y síntomas de la enfermedad, constatado en otros estudios (Miravitlles et al, 2006) y supone no sólo un problema de denominación, sino también de conciencia y asignación de entidad a la enfermedad como tal, con características propias, separada de otras enfermedades y diferenciada del efecto del envejecimiento.

En este hecho puede influir la falta de una imagen social de la EPOC al contrario que en otras enfermedades crónicas. La EPOC es muy poco conocida, confundiéndose con otras enfermedades respiratorias (Parker, Goldman y Eaton, 2008); no suele tener una historia "pública", y no se "espera" socialmente que se cure o que ocasione muerte, ni "esperan" padecer la enfermedad los pacientes que están en riesgo: las personas con hábito tabáquico prolongado perciben más riesgo de padecer cáncer o enfermedades cardiovasculares.

Respecto al diagnóstico, no es una enfermedad que se "busque" activamente: el estudio que lleva al diagnóstico no se inicia por presentar un hábito tabáquico intenso y/o prolongado, como se propone en las guías clínicas sobre EPOC (NICE, 2010); el paciente no suele pensar que la pueda padecer, no existiendo percepción del riesgo hasta la aparición de manifestaciones importantes (reagudizaciones, disnea severa). El diagnóstico en descompensaciones severas impide tener conciencia de enfermedad una vez recuperada esta fase. El hecho de diagnosticarla en el estudio por otros problemas (neumonías, apnea del sueño...) supone aumentar la confusión sobre los síntomas, sobre las causas de la enfermedad y sobre la evolución. El inicio de la enfermedad se produce en "un mar de confusiones" que se alimentan unas a otras.

En cuanto a las causas, la percepción de los pacientes sobre el papel de otros agentes diferentes al tabaco en la generación de la enfermedad tiene fundamento científico en algunos casos. Existen estudios (Balmes et al., 2003; Govender, Lalloo y Naidoo, 2011; Hnizdo, Sullivan, Bang y Warner, 2002) que avalan el efecto de la exposición profesional entre trabajadores de muy diversas ocupaciones (industrias plásticas, textiles, manufactura de productos alimentarios, construcción, agricultura, limpieza profesional) y que la asociación de esta exposición con el tabaquismo aumenta aún más el riesgo de desarrollar EPOC (Blanc et al., 2009).

La exposición continuada a productos de la combustión en ambientes cerrados también se ha relacionado con esta enfermedad (Orozco-Levi et al, 2006).

Aunque en algunos estudios se reconoce el posible papel de infecciones respiratorias (Hogg, 2004) que podrían actuar como coadyuvantes al efecto del tabaco, no se ha corroborado ninguna relación entre infecciones respiratorias de adultos y niños y génesis y evolución de la EPOC (SolerCataluña, 2007).

El "algo más" que el tabaco del que hablan los pacientes, hace referencia a causas sobre las que no tienen capacidad decisoria, externalizando la responsabilidad de adquirir la enfermedad. A diferencia de otras enfermedades, la EPOC se vincula a una adicción evitable que culpabilizaría socialmente a los pacientes. La importancia de la atribución causal se ve en estudios que muestran que los pacientes con EPOC que atribuyen la enfermedad a causas psicológicas e internas tienen peor ajuste emocional y peor estado funcional (Scharloo et al., 2007).

La diversidad en la percepción de los efectos de dejar de fumar puede relacionarse con el hecho de que el efecto de dejar de fumar es mayor en pacientes asintomáticos con afectación leve (Anthonisen, 2000) y que algunos enfermos no experimentan ningún cambio, independientemente del estadio de su EPOC (Pelkonen et al., 2000).

La importancia otorgada a la edad se refleja también en otras investigaciones cualitativas (Pinnock et al., 2011).

Respecto a la duración, en la EPOC, es difícil identificar un momento inicial de la enfermedad ya que el diagnóstico suele ser precedido de bastantes años de evolución lenta de los síntomas. En algunos casos existe un hito desencadenante del diagnóstico, que suele ser una infección y/o insuficiencia respiratoria severa pero no se establece una disrupción biográfica de la enfermedad, como en el caso de otras enfermedades crónicas.

Esta incertidumbre respecto al marco temporal de la enfermedad, afecta a la percepción de control de la persona que no tiene clara la evolución ni la duración (Vinaccia y Quiceno, 2011). 
Se identifica la clínica con las reagudizaciones y si entre ellas hay un largo intervalo temporal, la enfermedad parece "desaparecer".

La historia narrada de la enfermedad vista por el paciente que ya está en estadios avanzados, se describe como "caótica" (Pinnock et al., 2011), existiendo gran dificultad para separar la enfermedad de la historia vital y para construir un relato coherente diferenciado, como sí se consigue con otras enfermedades como el cáncer.

Respecto a las consecuencias, al igual que en nuestra investigación, se ha encontrado la infraestimación de la severidad de la enfermedad en otros estudios (Habraken, Pols, Bindels y Willems, 2008; Pitta et al., 2005; Rennard et al., 2008). El hecho de que los pacientes normalicen sus síntomas y solo se consideren enfermos en las reagudizaciones, puede considerarse "positivo": el efecto de prestar poca atención a los síntomas se relaciona con mejor calidad de vida autopercibida (Scharloo et al., 2007). Por otro lado, la infraestimación se produce al reducir su actividad física al nivel que evita la aparición de la disnea, y se favorece la pérdida de funcionalidad (Oga et al., 2005; Pitta et al., 2005). Esta reducción de actividad es especialmente intensa y prolongada durante y tras las reagudizaciones.

Respecto a la disnea, como en otros estudios, es un síntoma con considerable repercusión en el estilo de vida de los pacientes con EPOC (Reardon, Lareau y ZuWallack, 2006). La disnea es un fenómeno complejo en que los pacientes experimentan una mezcla de respuestas fisiológicas, psicológicas, medioambientales y conductuales, lo que debe tenerse en cuenta en las intervenciones orientadas a su control. Además aparece en fases avanzadas y en las reagudizaciones, y se percibe de forma desigual, sobre todo por los pacientes de mayor edad, debido a un efecto de adaptación del grado de actividad física para reducir los síntomas (Peces-Barba et al., 2008) y a la tendencia a relacionarlo con la edad. Además, la limitación que ocasiona la disnea se confunde con otros síntomas como la fatiga o cansancio.

Es llamativa la expresión de síntomas no respiratorios, menos "esperados" o considerados por los profesionales sanitarios: dolor, cansancio y mal estado general.

En otros estudios con pacientes EPOC (Vinaccia y Quiceno, 2011) se pone de manifiesto también la presencia de cansancio y agotamiento, que disminuyen los sentimientos de energía y vitalidad y la capacidad de realizar actividades. También es relevante el dolor corporal, que se relaciona con respuestas emocionales negativas.

En cuanto a las reagudizaciones, el miedo a padecerlas incluye el temor a la muerte y se aprecia cómo desaparece una vez pasado el episodio (Rennard et al., 2002). Este temor es fundado ya que las investigaciones demuestran cómo las exacerbaciones implican una pérdida acelerada de la función pulmonar, una progresión más rápida de la enfermedad y una disminución de la calidad de vida (Makris et al., 2007) siendo un elemento clave en su curso y pronóstico (Donaldson, Seemungal, Bohwmik y Wedzicha, 2002; Hurst et al., 2010). Un aspecto importante del temor a las reagudizacio- nes es la incertidumbre: no sabemos cuándo se van a presentar hasta que ya se manifiestan, se desconoce su gravedad o tipo, su duración ni sus consecuencias. Es así porque no se relacionan directamente con el estado basal del paciente y no siempre se dan en pacientes con mayor afectación ( $\mathrm{O}^{\prime}$ Really, Williams, Holt y Rice, 2006).

Son relevantes las repercusiones familiares y en el ocio señaladas por los participantes, ya que el aislamiento social y la reducción de actividades se asocia con afrontamiento inadaptativo pasivo a la enfermedad (Seamark, Blake y Seamark, 2004).

Respecto a los miedos, son similares a los encontrados en otros pacientes que llegan a considerar la EPOC peor que el cáncer (Gore, Brophya y Greenstonea, 2000). La oxigenoterapia también se señala como uno de los factores que más afectan a la CVRS (Carrasco et al., 2006), mediante varios mecanismos, que incluyen la expectativa negativa de depender de una máquina para mantener la salud, el ruido causado por el aparato, la restricción de la movilidad y el confinamiento en domicilio (Sant'Anna et al., 2003; Wedzicha, 2000). El temor al uso de los inhaladores aparece en otros estudios y se relaciona con el cese deliberado, temporal o definitivo del tratamiento (Gatti, Jacobson, Ganzmararian, Schomotzer y Kripalani, 2009).

También son llamativos los miedos que no aparecen: el temor a padecer cáncer de pulmón, que en otros estudios con pacientes en riesgo de o con EPOC en atención primaria, aparece como un temor relevante (Habraken et al ., 2008).

Considerando aspectos de control/curación de la enfermedad, la percepción de control sobre la EPOC es fundamental porque influencia de forma relevante el automanejo de ésta (Dowson, Town, Frampton y Mulder, 2004). Algunos autores proponen diferenciar entre autocontrol sobre los síntomas y autocontrol sobre mantener la funcionalidad, siendo este segundo aspecto predictivo de mejor calidad de vida en las esferas psicológica y social (Arnold et al., 2006).

Aparte del abandono del hábito tabáquico que constituye la intervención fundamental sobre la enfermedad, se sabe que un correcto tratamiento puede mantener e incluso mejorar la función pulmonar (Di Mateo, Haskard y Williams, 2007), en oposición al deterioro imparable que muestran los pacientes. La percepción de un curso inexorable de la enfermedad potenciaría la indefensión y baja autoeficacia de los pacientes. Este aspecto es importante para el control de la enfermedad ya que existe correlación positiva entre adherencia al tratamiento y percepción de la severidad de la enfermedad crónica (Di Mateo, 2007). No obstante, algunos pacientes mantienen una actitud activa, de búsqueda de mejoría en "cuidarse", que además depende de ellos mismos, reflejando un alta percepción de autoeficacia, que ha demostrado efectos beneficiosos (McCathie, Spence y Tate, 2002).

Respecto al tratamiento inhalado, la dificultad parece no estar tanto en la técnica, sino en las expectativas de su beneficio: el efecto real (broncodilatar) no es apreciable directa ni inmediatamente, el efecto deseado ("limpiar") sí lo sería. 
Además existe un dificultoso y tardío reconocimiento de los efectos, que diferencia la EPOC de otras enfermedades crónicas en que existen medidas objetivas de control por el propio paciente: medición de la presión arterial o de la glucemia en pacientes con hipertensión o diabetes. Las creencias erróneas respecto a los inhaladores, la percepción de efectos secundarios y las confusas pautas de tratamiento, serían factores añadidos que implicarían una importante dificultad para la adherencia terapéutica.

Las principales limitaciones de nuestro estudio se deben a la selección oportunista de los pacientes, aunque en el marco de un ensayo clínico aleatorizado, si bien los participantes se consideran informantes que muestran riqueza narrativa suficiente para los objetivos de la investigación. La entrevista grupal se ha realizado con bajo número de participantes por grupo, lo que ocasiona menos interacción aunque garantiza suficiente obtención de información, compensada con mayor número de grupos y criterios de saturación. Los grupos de validación se han realizado bastante después del grupo original lo que puede condicionar cambios en la visión de la enfermedad.

Respecto a la aplicación práctica de este estudio, la falta un modelo mental coherente sobre la EPOC, se traduce también en un desconcierto conductual: el modo en el que se representa la amenaza de la enfermedad para la vida promueve un plan de acción frente a la misma, la falta de esa representación impide una imagen de amenaza suficientemente clara para actuar en consecuencia.

Nuestros resultados manifiestan la necesidad de considerar la perspectiva de los pacientes sobre la enfermedad a la hora de planificar las intervenciones con cada persona en concreto y guiar la formación de un modelo mental de la EPOC menos confuso y más acorde a la mejor evidencia disponible.

\section{Referencias}

Álvarez-Gutiérrez, F.J., Miravitlles, M., Calle, M., Gobartt, E., López, F. y Martín, A.; Grupo de estudio EIME. (2007). Impact of Chronic Obstructive Pulmonary Disease on Activities of Daily Living: results of the Multicenter EIME Study. Archivos de Bronconeumología, 43, 64-72. Doi:10.1016/S1579-2129(07)60026-3

Anthonisen, N.R. (2000). Smoking, lung function and mortality. Thorax, 55, 729-734. Doi:10.1136/thorax.55.9.729

Arnold, R., Ranchor, A.V., Koëter, G.H., de Jongste, M.J., Wempe, J.B., ten Hacken, N.H., ... Sanderman, R. (2006). Changes in personal control as predictor of quality of life after pulmonary rehabilitation. Patient Education y Counseling, 61, 99-108. Doi:10.1016/i.pec.2005.02.015

Barnestein-Fonseca, P., Leiva-Fernández, J., Vidal-España, F., García-Ruiz, A., Prados-Torres, D. y Leiva-Fernández, F. (2011). Efficacy and safety of a multifactor intervention to improve therapeutic adherence in patients with chronic obstructive pulmonary disease (COPD): protocol for the ICEPOC study. Trials, 12, 40. Doi:10.1186/1745-6215-12-40

Balmes, J., Becklake, M., Blanc, P., Henneberger, P., Kreiss, K., Mapp, C., ... Viegi, J., Environmental and Occupational Health Assembly American Thoracic Society. (2003). American Thoracic Society statement: occupational contribution to the burden of airway disease. American Journal of Respiratory and Critical Care Medicine, 167, 787-797. Doi: $10.1164 / \mathrm{rccm} .167 .5 .787$
Se propondría la evaluación de la percepción de la enfermedad como parte de la valoración clínica de los pacientes con EPOC y la utilización de métodos de cambio de las percepciones erróneas como forma de mejorar las consecuencias de la enfermedad (Kaptein et al., 2008). Así, programas de reestructuración cognitiva han mostrado mejoras en capacidad funcional y bienestar de los pacientes y en el tratamiento de la ansiedad y depresión asociada (Kunik et al., 2008).

Este estudio pone de manifiesto la necesidad de difundir a los profesionales (de medicina y enfermería), las confusiones que tienen estos pacientes respecto a su proceso, para tomar conciencia de esta situación y dotarse de herramientas (empatía, exploración de creencias, adaptación de la información y recomendaciones a cada persona) que les ayuden a avanzar en un abordaje más acertado de estos pacientes.

Como conclusiones de nuestro estudio podemos resumir que la EPOC es una enfermedad poco percibida como tal por los pacientes que la padecen, existiendo importantes confusiones respecto al nombre e identidad y reconociéndose el papel del tabaco en su génesis aunque con dudas y fuerte intervención de otras causas. El patrón evolutivo se reconoce crónico con empeoramientos recurrentes y con importante confusión con los efectos de la edad y con otras enfermedades.

Los síntomas son frecuentemente reducidos por adaptación de la funcionalidad al nivel que impide su presencia. Los principales son respiratorios pero también no respiratorios como el dolor y el cansancio. Los sentimientos asociados son la desesperanza y el miedo al deterioro, a la dependencia del oxígeno, a los resfriados. Al tratamiento le piden “desatascar" y existe confusión y creencias erróneas sobre el efecto y pautas de utilización de los inhaladores, siendo muy importantes los aspectos sensoriales.

Blanc, P., Iribarren, C., Trupin, L., Earnest, G., Katz, P.P., Balmes, J., ... Eisner, M.D. (2009). Occupational exposures and the risk of COPD: dusty trades revisited. Thorax, 64, 6-12. Doi: 10.1136/thx.2008.099390

Boult, C. y Wieland, G.D. (2010). Comprehensive primary care for older patients with multiple chronic conditions. "Nobody rushes you through". Journal of the American Medical Association, 304, 1936-1943. Doi:10.1001/jama.2010.1623

Calleja, L.A. (2014). Cómo vive el paciente con EPOC las reagudizaciones. Estudio cualitativo en medio hospitalario. (Trabajo final de experto universitario en medicina de urgencias y emergencias). Universidad Internacional de Andalucía, Málaga. Recuperado de http://dspace.unia.es/bitstream/handle/10334/2728/0546_Calleja.pdf ?sequence $=3$

Cameron, L. y Leventhal, H. (2003). The Self-regulation of Health and Illness Behaviour. London, England: Routledge.

Carrasco, P., de Miguel, J., Rejas, J., Martín, A., Gobartt, E., Gil, A., ... Jiménez, R. (2006). Negative impact of chronic obstructive pulmonary disease on the health-related quality of life of patients. Results of the EPIDEPOC study. Health and Quality of Life Outcomes, 4, 31. Doi:10.1186/1477-7525-4-31

De la Iglesia, F., de la Fuente, R., Ramos, V., Pellicer, C., Nicolás, R. y DizLois, F. (2001). Análisis factorial de la calidad de vida relacionada con la salud de los pacientes con enfermedad pulmonar obstructiva crónica es- 
table. Archivos de Bronconeumología, 37, 410-416. Recuperado de http://www.archbronconeumol.org/es/

De Miguel, J., Izquierdo, J.L., Rodríguez, J.M., De Lucas, P., Bellón, J.M. y Molina, J. (2004). Calidad de vida en la enfermedad pulmonar obstructiva crónica. Influencia del nivel de asistencia de los pacientes. Archivos de Bronconeumología, 40, 431-437. Doi: 10.1157/13066499

DiMatteo, M.R., Haskard, K.B. y Williams, S.L. (2007). Health beliefs, disease severity and patient adherence: a meta-analysis. Medical Care, 45, 521-528. Doi:10.1097/MLR.0b013e318032937e

Dirección General de Bienestar Social, Consejería para la Igualdad y Bienestar Social. (2006). Intervención coordinada de la Junta de Andalucía en zonas con necesidades de transformación social. Junta de Andalucía. Recuperado de http://www.juntadeandalucia.es/salud/servicios/contenidos/andalucia essalud/doc/Intervencion.pdf

Donaldson, G.C., Seemungal, T.A.R., Bohwmik, A. y Wedzicha JA. (2002). Relationship between exacerbation frequency and lung function decline in chronic obstructive pulmonary disease. Thorax, 57, 847-852. Doi:10.1136/thorax.57.10.847

Dowson, C.A., Town, G.I., Frampton, C. y Mulder, R.T. (2004). Psychopatology and illness beliefs influence COPD self-management. Journal of Psychosomatic Research, 56, 333-340. Doi: 10.1016/S0022-3999(03)00040-0

Ferrer, M., Alonso, J., Prieto, L., Plaza, V., Monsó, E., Marrades, R., ..., Antó J.M. (1996). Validity and reliability of the St George's Respiratory Questionnaire after adaptation to a different language and culture: the Spanish example. European Respiratory Journal, 9, 1160-1166. Doi: 10.1183/09031936.96.09061160

Frostholm, L., Fink, P., Christensen, K.S., Toft, T., Oernboel, E., Olesen, F. y Weinman, J. (2005). The patients' illness perceptions and the use of primary health care. Psychosomatic Medicine, 67, 997-1005. Doi:10.1097/01.psy.0000189164.85653.bc

Frostholm, L., Fink, P., Oernboel, E., Christensen, K.S., Toft, T., Olesen, F. y Weinman, J. (2005). The uncertain consultation and patient satisfaction: the impact of patients' illness perceptions and a randomised controlled trial on the training of physicians' communication skills. Psychosomatic Medicine, 67, 897-905. Recuperado de http://journals.lww.com/psychosomaticmedicine/pages/default.aspx

Gatti, M.E., Jacobson, K.L., Gazmararian, J.A., Schmotzer, B. y Kripalani, S. (2009). Relationship between beliefs about medications and adherence. American Journal of Health-System Pharmacy, 66, 657-664. Doi: 10.2146/ajhp080064

Gore, J.M., Brophy, C.J. y Greenstone, M.A. (2000). How well do we care for patients with end-stage chronic obstructive pulmonary disease (COPD)? A comparison of palliative care and quality of life in COPD and lung cancer. Thorax, 55, 1000-1006. Doi:10.1136/thorax.55.12.1000

Govender, N., Lalloo, U.G. y Naidoo, R.N. (2011). Occupational exposures and chronic obstructive pulmonary disease: a hospital based casecontrol study. Thorax, 66, 597-601. Doi:10.1136/thx.2010.149468

Habraken, J.M., Pols, J., Bindels, P.J.E. y Willems, D.L. (2008). The silence of patient with end-stage COPD: a qualitative study. British Journal of General Practice, 58, 844-849. Doi: 10.3399/bjgp08X376186

Hagger, M. (2003). A meta-analytic review of the common-sense model of illness representations. Psychology and Health, 18, 141-184. Doi:10.1080/088704403100081321

Han, M.K., Agusti, A., Calverley, P.M., Celli, B.R., Criner, G., Curtis, J.L., ... Martinez, F.J. (2010). Chronic obstructive pulmonary disease phenotypes: The future of COPD. American Journal of Respiratory and Critical Care Medicine, 182, 598-604. Doi: 10.1164/rccm.200912-1843CC

Hermele, S., Olivo, E.L., Namerow, O. y Oz, M.C. (2007). Illness representations and psychological distress in patients undergoing coronary artery bypass graft surgery. Psychology, Health y Medicine, 12, 580-591. Doi: 10.1080/13548500601162705

Hnizdo, E., Sullivan, P.A., Bang, K.M. y Wagner, G. (2002). Association between chronic obstructive pulmonary disease and employment by industry and occupation in the US population: a study of data from the Third National Health and Nutrition Examination Survey. American Journal of Epidemiology, 156, 738-746. Doi: 10.1093/aje/kwf105

Hogg, J.C. (2004). Pathophysiology of airflow limitation in chronic obstructive pulmonary disease. The Lancet , 364, 709-721. Doi: 10.1016/S01406736(04)16900-6
Hurst, J.R., Vestbo, J., Anzueto, A., Locantore, N., Müllerova, H., TalSinger, R., ... Wedzicha J.A. (2010) Suceptibility to Exacerbation in Chronic Obstructive Pulmonary Disease. New England Journal of Medicine, 363, 1128-1138. Doi: 10.1056/NEJMoa0909883

Jefferson, G. (1985). An exercise in the transcription and analysis of laughter. In: Van Dijk, T.A. (Ed.), Handbook of discourse analysis (pp. 25-34). London, England: Academic Press.

Johnson-Laird, P.N. (1983). Mental Models: towards a cognitive science of language, inference and conscientiousness, Cambridge, MA: Harvard University Press.

Jordan, N., Lee, T.A., Valenstein, M., Pirraglia, P. A. y Weiss KB. (2009). Effect of depression care on outcomes in COPD patients with depression. Chest, 35, 626-632. Doi: 10.1378/chest.08-0839

Kaptein, A.A., Scharloo, M., Fischer, M.J., Snoei, L., Cameron, L.D., Sont, J.K., Rabe K.F., ...Weinman, J. (2008). Illness perceptions and COPD: An emerging field for COPD patient management. Journal of Asthma, 45 625-629. Doi: 10.1080/02770900802127048

Kelly, C. y Lynes, D. (2008). Psychological effects of chronic lung disease. Nursing Times, 104, 82-85. Recuperado de http://www.nursingtimes.net

Kohler, C.L., Fish, L. y Greene, P.G. (2002). The relationship of perceived self-efficacy to quality of life in Chronic Obstructive Pulmonary Disease. Health Psychology, 21, 610-614. Doi: 10.1037/0278-6133.21.6.610

Kunik, M.E., Veazey, C., Cully, J.A., Souchek, J., Graham, D.P., Hopko, D, ... Stanley, M.A. (2008). . COPD education and cognitive behavioral therapy group treatment for clinically significant symptoms of depression and anxiety in COPD patients: a randomised controlled trial. Psychological Medicine, 38, 385-396. Doi: 10.1017/S0033291707001687

Leventhal, H., Meyer, D., y Nerenz, D.R. (1980). The common sense representation of illness danger. In: Rachman, S., (Ed), Contributions to Medical Psychology (pp. 17-30). New York, NY: Pergamon Press.

Maillé, A.R., Koning, C.J.M., Zwinderman, A.H., Willems, L.N.A., Dijkman, J.H. y Kaptein, A.A. (1997). The development of the "Quality of life for respiratory illness questionnaire (QOL-RIQ): a disease-specific quality of life questionnaire for patients with mild to moderate chronic nonspecific lung disease. Respiratory Medicine, 91, 297-309. Doi: 10.1016/S0954-6111(97)90034-2

Makris, D., Moschandreas, J., Damianaki, A., Ntaoukakis, E., Siafakas, N.M., Milic, E.J. y Tzanakis, N. (2007). Exacerbations and lung function decline in COPD: New insights in current and ex-smokers. Respiratory Medicine, 101,1305-1312. Doi: 10.1016/j.rmed.2006.10.012

Maurer, J., Rebbapragada, V., Borson, S., Goldstein, R., Kunik, M.E., Yohanes, A.M. y Hanania, N.A.; ACCP Workshop Panel on Anxiety and depression in COPD. (2008). Anxiety and depression in COPD: current understanding, unanswered questions and research needs. Chest, 134, 44-56. Doi: 10.1378/chest.08-0342

McCathie, H.C., Spence, S.H. y Tate, R.L. (2002). Adjustment to chronic obstructive pulmonary disease: the importance of psychological factors. European Respiratory Journal, 19, 47-53. Doi: 10.1183/09031936.02.00240702

Medinas, M.M., Más, C. y Renom, F. (2009). Estrés en ancianos hospitalizados con enfermedad respiratoria crónica: Valoración en el hospital sociosanitario. Revista Española de Geriatría y Gerontología, 44, 130-136. Doi: 10.1016/j.regg.2009.01.006

Miravitlles, M., Anzueto, A., Legnani, D., Forstmeier, L. y Fargel, M. (2007). Patient's perception of exacerbation of COPD. The PERCEIVE study. Respiratory Medicine, 101, 453-460. Doi: 10.1016/j.rmed.2006.07.010

Miravitlles, M., de la Roza, C., Morera, J., Montemayor, T., Gobartt, E., Martín, A. y Alvarez-Sala, J.L. (2006). Chronic respiratory symptoms, spirometry and knowledge of COPD among general population. Respiratory Medicine, 100, 1973-1980. Doi: 10.1016/i.rmed.2006.02.024

Miravitlles, M., Soriano, J.B., García-Río, F., Muñoz, L., Duran-Tauleria, E., Sánchez, G., ... Ancochea, J. (2009). Prevalence of COPD in Spain: impact of undiagnosed COPD on quality of life and daily life activities. Thorax, 64, 863-868. Doi: 10.1136/thx.2009.115725

Mousing, C.A. y Lomborg, K. (2012). Self-care 3 months after attending chronic obstructive pulmonary disease patient education: a qualitative descriptive analysis. Patient Preference and Adherence, 6, 19-25. Doi: 10.2147/PPA.S28466

National Institute for Health and Clinical Excellence. (2010). Chronic obstructive pulmonary disease: management of chronic obstructive pul- 
monary disease in adults in primary and secondary care. Recuperado de: http://guidance.nice.org.uk/CG101

Oga, T., Nishimura, K., Tsukino, M., Sato, S., Hajiro, T. y Mishima, M. (2005). Exercise capacity deterioration in patients with COPD: longitudinal evaluation over 5 years. Chest, 128, 62-69. Doi: 10.1378/chest.128.1.62

O'Reilly, J.F., Williams, A.E., Holt, K. y Rice, L. (2006). Defining COPD exacerbations: impact on estimation of incidence and burden in primary care. Primary Care Respiratory Journal, 15, 346-353. Doi: 10.1016/i.pcri.2006.08.009

Orozco-Levi, M., García-Aymerich, J., Villar, J., Ramírez-Sarmiento, A., Antó, J.M. y Gea, J. (2006). Wood smoke exposure and risk of chronic obstructive pulmonary disease. European Respiratory Journal, 27, 542-546. Doi:10.1183/09031936.06.00052705

Pacheco-Huergo, V., Viladrich, C., Pujol-Ribera, E., Cabezas-Peña, C., Núñez, M., Roura-Olmeda, P., ... del Val, J.L. (2012). Percepción en enfermedades crónicas: validación lingüística del Illness Perception Questionnaire Revised y del Brief Illness Perception Questionnaire para la población española. Atención Primaria, 44, 280-287. Doi: 10.1016/j.aprim.2010.11.022

Parker, D.R., Goldman, R.E. y Eaton, C.D. (2008). A qualitative study of individual at risk for or who have chronic obstructive pulmonary disease: what do they understand about their disease? Lung, 186, 313-316. Doi: 10.1007/s00408-008-9091-9

Peces-Barba, G., Barberá, J.A., Agustí, A., Casanova, C., Casas, A., Izquierdo, J.L., ... Viejo, J.L. (2008). Guía Clínica SEPAR-ALAT de diagnóstico y tratamiento de la EPOC. Archivos de Bronconeumología, 44, 271-281. Doi: $10.1157 / 13119943$ :

Pelkonen, M., Tukiainen, H., Tervahauta, M., Notkola, I.L., Kivelä, S.L., Salorinne, Y. y Nissinen, A. (2000). Pulmonary function, smoking cessation and 30 year mortality in middle aged Finnish men. Thorax, 55, 746750. Doi: 10.1136/thorax.55.9.746

Pinnock, H., Kendall, M., Murray, S., Worth, A., Levack, P., Porter, M., ...Sheikh A. (2011). Living and dying with severe chronic obstructive pulmonary disease: multi-perspective longitudinal qualitative study. British Medical Journal, 342, d142. Doi: 10.1136/bmj.d142

Pitta, F., Troosters, T., Spruit, M.A., Probst, V.S., Decramer, M. y Gosselink, R. (2005). Characteristics of physical activities in daily life in chronic obstructive pulmonary disease. American Journal of Respiratory and Critical Care Medicine, 171, 972-977. Doi: 10.1164/rccm.200407-855OC
Quiceno, J.M., Vinaccia, S. (2010). Percepción de enfermedad: una aproximación a partir del Illness Perception Questionnaire. Psicología desde El Caribe, 25, 56-83.

Reardon, J.Z., Lareau, S.C. y ZuWallack, R. (2006). Functional Status and quality of life in Chronic Obstructive Pulmonary Disease. American Journal of Medicine, 119, S32-S37. Doi: 10.1016/i.amimed.2006.08.005

Rennard, S., Decramer, M, Calverley, P.M.A., Pride, N.B., Soriano, J.B., Vermeire, P.A. y Vestbo, J. (2002). Impact of COPD in North America and Europe in 2000: subjects perspective of confronting COPD International Survey. European Respiratory Journal, 20, 799-805. Doi: 10.1183/09031936.02.03242002

Rennard, S.I. y Vestbo, J. (2008). The many “small COPDs": COPD should be an orphan disease. Chest, 134, 623-637. Doi: 10.1378/chest.07-3059

Scharloo, M., Kaptein, A.A., Schlösser, M., Pouwels, H., Bel, E.H., Rabe, K.F. y Wouters, E.F.M. (2007). Illness perceptions and quality of life in patients with Chronic Obstructive Pulmonary Disease. Journal of Asthma, 44, 575-581. Doi: 10.1080/02770900701537438

Sant'Anna, C.A., Stelmach, R., Zanetti, M.I., Filho, W.J., Chiba, T. y Cukier, A. (2003). Evaluation of health-related quality of life in low-income patients with COPD receiving long-term oxygen therapy. Chest, 123, 136141. doi:10.1378/chest.123.1.136

Seamark, D.A., Blake, S.D. y Seamark, C.J. (2004). Living with severe chronic obstructive pulmonary disease (COPD): Perceptions of patients and their carers. Palliative Medicine, 18, 619-625. Doi: $10.1191 / 0269216304 \mathrm{pm} 928 \mathrm{oa}$

Soler-Cataluña, J.J. (2007). The role played by exacerbations in the natural history of Chronic Obstructive Pulmonary Disease. Archivos de Bronconeumología, 43, 55-58. Doi: 10.1016/S1579-2129(07)60024-X

Soler-Cataluña, J.J., Martínez-García, M.A y, Catalán, P. (2010). Impacto multidimensional de las exacerbaciones de la EPOC. Archivos de Bronconeumología, 46 (Supl 11), 12-19. Doi: 10.1016/S0300-2896(10)70057-0

Vinaccia, S. y Quiceno, JM. (2011). Calidad de vida relacionada con la salud y factores psicológicos: un estudio desde la Enfermedad Pulmonar Obstructiva Crónica. Terapia Psicológica , 29, 65-75. Doi: 10.4067/S071848082011000100007

Wedzicha, J.A. (2000). Effects of long-term oxygen therapy on neuropsychiatric function and quality of life. Respiratory Care, 45, 119-124

(Artículo recibido: 19-11-2013; revisado: 15-01-2015; aceptado: 08-03-2015) 